Erschienen in: Deppermann, Arnulf/Linke, Angelika (Hrsg.): Sprache intermedial. Stimme und Schrift, Bild und Ton. - Berlin/New York: de Gruyter, 2010. S. 419-445. (Jahrbuch des Instituts für Deutsche Sprache 2009), https://doi.org/10.1515/9783110223613.419

Jannis Androutsopoulos

\title{
Multimodal - intertextuell - heteroglossisch: Sprach-Gestalten in „Web 2.0“-Umgebungen
}

\begin{abstract}
Web-Umgebungen wie virtuelle soziale Netzwerke und Videoportale sind von Tendenzen der Partizipation, Konvergenz und Multimedialität gekennzeichnet. Diese bedeuten eine Herausforderung für sprachanalytische Zugänge, die digitale Kommunikationsformen separat voneinander untersuchen und auf mikrolinguistische Phänomene bei nur geringer Beachtung ihrer komplexen soziomedialen Rahmenbedingungen abheben. Im Beitrag wird ein bildschirm-basierter Ansatz entworfen, der Web-Umgebungen als semiotische Räume begreift, die von Nutzern in ihren spezifischen soziokulturellen Umständen und vor der Folie technologischer Potenziale und Grenzen aufgefüllt und ausgestaltet werden. Sprache ist eine wesentliche, aber nicht die einzige Ressource dieses digitalen kommunikativen Handelns, und ihre Verwendung ist in der Spannung zwischen technologischer Vorprägung und situierter Medienaneignung zu untersuchen. Im Kern des Ansatzes liegt die Unterscheidung von zwei analytischen Dimensionen. Die erste unterscheidet vier Leistungen von Sprache in Web-Umgebungen: Organisation, Selbstdarstellung, Spektakel und Interaktion. Die zweite erfasst drei für Web 2.0 charakteristische Prozesse der Sprach- und Textgestaltung: Multimodalität, Intertextualität und Heteroglossie. Wie diese beiden Kategorienbündel eine Grundlage für weiterführende Fragestellungen bilden können, wird am Beispiel des Dialektgebrauchs auf einer Videoplattform diskutiert.
\end{abstract}

\section{Einleitung}

Trotz ihrer Bedeutung in der Öffentlichkeit und Alltagskultur haben gegenwärtige Entwicklungen in der Netzkommunikation, die oft mit dem Stichwort „Web 2.0“ belegt werden, aus linguistischer Sicht noch kaum Beachtung gefunden. Ziel dieses Beitrags ${ }^{1}$ ist es, Rahmenbedingungen für die Untersuchung von Web 2.0-Umgebungen zu erörtern und einen Analyserahmen zu skizzieren bzw. exemplarisch anzuwenden. Der Beitrag gliedert sich in drei aufeinander aufbauende Teile. In einem ersten Schritt diskutiere ich Kennzeichen von Web 2.0, hebe die Bedeutung von Partizipation und Konvergenz für kommunikatives Handeln hervor und gehe der Frage nach, wie die linguistische Internetforschung auf diese Entwicklungen reagieren kann. Im Kern des Beitrags liegt der Umriss eines bildschirm-basierten Zugangs zu Web 2.0-Umgebungen. Dieser umfasst zwei Kategorien-Sets, die als Antworten auf zwei grundlegende Fragen verstanden werden können: Erstens, wie trägt Sprache zur Konstitution gegenwärtiger Web-Umgebun-

1 Der Vortragsstil wurde weitgehend beibehalten. 
gen bei? Zweitens, welche Diskursprozesse kennzeichnen diese Umgebungen? Die analytische Ausfächerung dieser Kategorien und deren Querbezüge sollen einen deskriptiven Rahmen abstecken, in dem Innovationen gegenwärtiger Netzkommunikation genauso ihren Platz finden wie Traditionen der linguistischen Internetforschung. Anschließend wird an einem Fallbeispiel exemplarisch vorgeführt, wie die beiden Kategoriensets empirisch ineinander greifen und analytisch eingesetzt werden können. Viele der nachfolgend diskutierten Phänomene sind nicht völlig neu, sondern vielmehr als Fortsetzung, teilweise auch Potenzierung bestehender Muster und Tendenzen zu verstehen. Zudem geht es mir weniger um eine erschöpfende Erläuterung als um die programmatische Skizze, die exemplarische Erfassung ihrer Facetten und ihrer Zusammenwirkung.

\section{Das „soziale Netz“ und seine Kennzeichen}

Das Schlagwort „Web 2.0“ hat in den letzten Jahren wie fast kein anderes das öffentliche Verständnis von Entwicklungen in der Netzkommunikation geprägt. Dabei ist Web 2.0 kein Fachterminus, sondern ein vager, assoziationsreicher Begriff, der einer präzisen Definition entbehrt und nach Ansicht vieler Beobachter für den Fachdiskurs eher ungeeignet ist (Scholz 2008; Maaß/Pietsch 2007; Ebersbach/Glaser/Heigl 2008, S. 23; Beiträge in Zimmer 2008). Wenn er hier dennoch Verwendung finden soll, dann einerseits mit der gebührenden Distanz und aus einer kritischen Metaperspektive, andererseits aber, weil er den Anschluss linguistischer Überlegungen an interdisziplinäre und öffentliche Diskurse über Technologie, Gesellschaft und Kommunikation signalisiert.

Seit seiner Prägung (O'Reiley 2005) wird der Begriff an Merkmalslisten veranschaulicht. Wikipedia zum Beispiel schlägt folgende „characteristics of Web 2.0" vor: „rich user experience, user participation, dynamic content, metadata, web standards, scalability" (en.wikipedia.org 2009), während ein anderer Beobachter folgende „Key Aspects of Web 2.0“ unterscheidet (Hinchcliffe 2006):

- The Web and all its connected devices as one global platform of reusable services and data

- Data consumption and remixing from all sources, particularly user generated data

- Continuous and seamless update of software and data, often very rapidly

- Rich and interactive user interfaces

- Architecture of participation that encourages user contribution

Allerdings neigen solche Listen dazu, technologische (etwa: metadata) und gesellschaftliche (z.B. rich user experience) Aspekte des Netzes nebeneinander zu stellen, ohne deren wechselseitiges Verhältnis zu berücksichtigen. Eine 
zweite und meines Erachtens einleuchtendere Begriffserläuterung ist die zugespitzte Gegenüberstellung zwischen Web 2.0 und „Web 1.0“, einer retrospektiv geprägten Bezeichnung für das frühe Netz der 1990er Jahre (O'Reiley 2005). Demnach bestand Web 1.0 aus „traditionellen“ Websites, die nur von ihren Besitzern bzw. Produzenten verändert werden konnten, während die Nutzer ${ }^{2}$ auf die Rezeption der bereitgestellten Inhalte eingeschränkt waren. Die interaktiven Anwendungen des Internet wie Email, Newsgroups, Mailinglisten und Chatkanäle, die das ursprüngliche Hauptgebiet der linguistischen Internetforschung darstellten, operierten noch separat vom Web 1.0 und gingen ihm historisch voraus. Die Unterscheidung zwischen dem Web als Informationsquelle (,Web 1.0“) und den interaktiven Internet-Diensten war noch bis zu Beginn dieses Jahrzehnts allgemein üblich.

Eben diese Unterscheidung fällt nun mit Web 2.0 in sich zusammen. Sein erstes Kennzeichen, Partizipation, ist wesentlich weiter aufgefasst als interpersonale Kommunikation im sprachwissenschaftlichen Sinn. Web 2.0Umgebungen sind keine Ansammlungen redaktionell aufbereiteter Inhalte, sondern stellen Infrastrukturen bereit, die von Nutzern - freilich bei entsprechender Hardware-Ausstattung und passendem Breitband-Zugriff rezeptiv und produktiv angeeignet werden. Innerhalb der von der jeweiligen Plattform gesetzten thematischen, technischen und semiotischen Grenzen werden Inhalte von den Nutzern selbst beigesteuert - hochgeladen, modifiziert, kommentiert, mit anderen Inhalten verknüpft usw. Dadurch „kann jeder durchschnittlich befähigte Nutzer, selbst wenn er nicht programmieren kann, viel leichter als bisher aktiv an der Informations- und Meinungsverbreitung teilnehmen“ - das Motto lautet: „Jeder kann mitmachen“ (de. wikipedia.org 2009). In diesem Sinne ist Web 2.0 durch eine „Architektur des Mitwirkens" (Hinchcliffe 2006) gekennzeichnet; von Randow (2007) bezeichnet es als „Medium der Beteiligung“:

Das Web 2.0 ist ein Medium der Beteiligung. (...) Der Erfolg von Angeboten wie YouTube oder MySpace rührt nicht zuletzt daher, dass hier jedermann sein Zeug raufladen kann, um wenigstens einmal Prominenz zu erfahren, Mikroprominenz. (Randow 2007)

Dem Beteiligungsprinzip kommen die Stichworte „Plattform“ und „Umgebung" entgegen, wie sie in diesem Beitrag verwendet werden. Dabei ist mit „Umgebung“ ein semiotischer Raum gemeint, in dem Nutzer verschiedene Handlungsmöglichkeiten zur Verfügung haben, während „Plattform“ eine spezifische technisch-institutionelle Basis für die Verwendung digitaler Kommunikationstechnologien meint. So stellen virtuelle soziale Netzwerke vielschichtige Kommunikationsumgebungen dar, jedes einzelne davon ist eine spezifische Vernetzungsplattform mit eigenen Partizipationsbedingungen und Gestaltungskonventionen.

Ich verwende das generische Maskulinum. 
Das Prinzip der Beteiligung tritt auf den unter Web 2.0. zusammengefassten Umgebungen auf unterschiedliche Weise in Erscheinung. Im Folgenden sollen nur zwei Umgebungstypen Berücksichtigung finden: Vernetzungs- und Medienplattformen.

Vernetzungsplattformen (andere gängige Bezeichnungen sind „(virtuelle) soziale Netzwerke“, „Social-Network-Dienste“, „, social network sites") ermöglichen ihren Nutzern die Einrichtung einer Profilseite und die Vernetzung mit anderen Profilseiten. Facebook und MySpace sind die international wohl bekanntesten Plattformen dieser Art, zu ihnen gesellen sich eine Vielzahl anderer, darunter auch regional- bzw. sprachspezifischer Varianten. Nach boyd (2008) weisen social network sites drei distinktive Merkmale auf: a) Profile - individuelle „virtuelle Körper“, die unter Zuhilfenahme der jeweils verfügbaren semiotischen Ressourcen gestaltet werden; b) Freunde - die auf der Plattform sichtbar gemachten sozialen Beziehungen; und c) Kommentare - Beiträge, die „Freunde“ auf den Profilseiten anderer „Freunde“ hinterlassen. ${ }^{3}$

Mediaplattformen (auch content sharing sites, media sharing sites oder social sharing genannt) dienen der Bereitstellung digitaler Medientexte wie Fotos, Videos und Musik für eine meist unbegrenzte Netzöffentlichkeit. Von der Produktionsseite her weisen Mediaplattformen wie flicker und YouTube eine Bandbreite privater, kommerzieller und institutioneller Nutzung auf, wobei im Folgenden nur der private Bereich interessiert. In Analogie zu den Vernetzungs- lassen sich auch für Mediaplattformen drei Kennzeichen bestimmen: a) Präsentation jeder Medieneinheit auf einer dezidierten Webseite, b) Nutzungsstatistiken wie Aufrufe, Bewertungen und Herkunft der Besucher und c) eine Kommentierungsmöglichkeit.

Eine zweite sprach- und diskursanalytisch relevante Dimension der gegenwärtigen Netzentwicklung, die in den erwähnten Merkmalsauflistungen weniger deutlich zum Ausdruck kommt, wird unter dem Dachbegriff Konvergenz zusammengefasst. Damit ist allgemein die Zusammenführung, Verflechtung und Verschmelzung von technischen und kommunikativen Prozessen und Aktivitäten der Medienproduktion bzw. Medienrezeption gemeint. In der medienwissenschaftlichen Diskussion wird Konvergenz verschiedenartig definiert und analytisch ausgelegt (vgl. Jenkins 2006; Hasebrink/Mikos/Prommer (Hg.) 2004). Für meine Zwecke unterscheide ich in Bezug auf Netzkommunikation drei Teilaspekte von Konvergenz: Integration, Einbettung und Modularität.

\footnotetext{
Soziale Netzwerke bedeuten nach boyd (2008) einen Paradigmenwechsel weg von den viel beachteten und untersuchten virtuellen Gemeinschaften. Das regelmäßige virtuelle „Miteinander" von Gleichgesinnten auf einer spezifischen Plattform (Chat- oder Forumsgemeinschaften) wird abgelöst durch losere, heterogenere Ansammlungen von „Individuen“, deren Gemeinschaftlichkeit nicht erst online, sondern in erster Linie offline entsteht; dieser Unterscheid dürfte auch auf die linguistische Beschäftigung mit dem Netz Auswirkungen haben (vgl. etwa die Operationalisierung von virtuellen Gemeinschaften bei Herring 2004).
} 
Mit „Integration“ ist gemeint, dass ehemals separate digitale Kommunikationsformen auf das Web übertragen und innerhalb ein und derselben Plattform ausgeführt werden. Beispielsweise bietet die Vernetzungsplattform facebook eine Reihe von Kommunikationsmöglichkeiten, die traditionellen Internet-Diensten ähneln bzw. diese integrieren: die persönlichen Nachrichten sind vergleichbar zu Emails, die „Chat"-Funktion ist im Grunde ein Instant Messenger für den individuellen quasi-synchronen Austausch; Beiträge auf der „Wand“ eines „Freundes“ ähneln den früheren Gästebucheinträgen auf einer persönlichen Homepage, und Gruppendiskussionen sind das strukturelle Äquivalent zu Newsgroups oder Webforen. Insofern sprengen gegenwärtige Vernetzungsplattformen frühere Klassifizierungen digitaler Kommunikationsformen (etwa nach Herring 2001 oder Döring 2003), da sie mehrere Zellen auf einmal belegen. „Einbettung“ meint die Möglichkeit, digitale Texte auf nahezu beliebige Weise zu vervielfältigen und auf Webseiten zu platzieren; so lassen sich beispielsweise YouTubeVideos auf eine Blog- oder Profilseite einbetten, wo sie weiter kommentiert werden können. Durch Einbettung werden der Umlauf und die Kombinierbarkeit digitaler Inhalte potenziert. „Modularität“ meint schließlich die Zusammensetzung von Webseiten aus separaten Textelementen („Modulen"), die auch in anderen Kombinationen auftreten können. Eine Spielart von Modularität ist das als Mashup bekannte Verfahren, wodurch eine individuelle Seite aus Inhalten aus verschiedenen Quellen collagenartig zusammengestellt wird; dies entspricht dem Web 2.0-Kennzeichen „Data consumption and remixing from all sources" (nach Hinchcliffe 2006).

Zieht man die skizzierten Prozesse zusammen, so erscheint Web 2.0 als ein Entwicklungsmoment der „Neuen Medien“, das von der Koexistenz unterschiedlicher, teilweise gegensätzlicher Elemente und Prozesse gekennzeichnet ist: Privates und Öffentliches, Professionelles und Amateurhaftes, Globales und Lokales kommen auf engstem Raum, auf ein und derselben Plattform oder sogar Webseite, in neuartigen Kombinationen zusammen.

\section{Web 2.0 vor der Folie der linguistischen Internetforschung}

Partizipation und Konvergenz sind nun im Hinblick auf ihre sprachlichdiskursiven Auswirkungen und Implikationen zu bedenken. Gleich an dieser Stelle sei angemerkt, dass das Verhältnis zwischen medien- und sprachwissenschaftlichen Perspektiven auf Web 2.0 sich nicht auf einen direkten disziplinären Transfer von Kategorien und Kriterien erschöpft. Die Tatsache, dass manche der bisherigen Beobachtungen und Typologien sprachanalytisch anschlussfähig erscheinen, bedeutet noch nicht, dass gesamte Merkmalslisten wie die eingangs zitierten übernommen und linguistisch be- 
legt werden könnten. Außerdem mag eine sprachwissenschaftliche Perspektive auf Web 2.0 auch Tendenzen aufspüren und Relevanzen setzen, die aus Sicht anderer Disziplinen weniger interessant oder sichtbar sind.

In diesem Sinne ist zunächst zu konstatieren, dass das Stichwort „Partizipation" mit unabhängig belegten Tendenzen des digitalen Sprachgebrauchs sehr gut vereinbar ist. Durch Partizipation - und das heißt: durch die außerinstitutionelle, nichtprofessionelle Produktion öffentlich zugänglicher Texte - kann sich der Anteil informeller schriftlicher Kommunikationsstile im öffentlichen Netzraum noch weiter erhöhen und verfestigen. Dies schließt den Einzug mündlichkeitsnaher Schreibstile in herkömmliche Domänen konzeptioneller Schriftlichkeit sowie die erhöhte Sichtbarkeit von Nonstandardvarietäten mit ein. Partizipation und Konvergenz bewirken, dass Rollentrennungen zwischen Produzenten/Informationsanbietern und Rezipienten/Konsumenten zunehmend aufgelockert und Grenzen zwischen unterschiedlichen Diskurstypen, Gattungen und Schreibstilen zunehmend verwischt werden. Institutionell produzierte und Nutzer generierte Inhalte können nun nebeneinander erscheinen und neue Kombinationen eingehen - ein Prozess, der beispielsweise beim Besuch von Bewertungsplattformen oder Onlineshops, die Kundenrezensionen einbinden, leicht erkennbar ist. Durch Konvergenz wird zudem die Komplexität der semiotischen Zusammensetzung digitaler Umgebungen erhöht. Die Teilaspekte der Einbettung und Modularität bedeuten, dass einzelne Webseiten aus Elementen ganz verschiedener Herkunft - und damit auch potenziell uneinheitlicher Autorenschaft, Gattung und Stillage - zusammengesetzt sind.

Die künftige Auseinandersetzung linguistischer Forschung mit diesen Prozessen ${ }^{4}$ wird auf eine inzwischen reichhaltige Forschungslage zurückgreifen können, die es ermöglicht, Web 2.0 nicht als ein vermeintlich völlig neues Phänomen zu begreifen, sondern seine innovativen Facetten vor der Folie früherer Stadien von Netzkommunikation einzuschätzen. Andererseits werden sich dabei nicht alle bisherigen Ansätze als gleichermaßen produktiv und anschlussfähig erweisen. An dieser Stelle sei insbesondere auf zwei Forschungsprämissen aufmerksam gemacht, die von den gegenwärtigen Entwicklungen meines Erachtens in Frage gestellt werden: die analytische Trennung von Kommunikationsformen und die Dekontextualisierung sprachlicher Daten.

Damit ist erstens die verbreitete Praxis gemeint, Sprachgebrauch im Internet separat für einzelne Kommunikationsformen zu untersuchen und als Ergebnis Listen von vermeintlich anwendungsspezifischen Sprach- und Stilmitteln aufzustellen. Dadurch haben sich die einzelnen digitalen Kommu-

Beiträge zu Web 2.0-Umgebungen aus spezifisch sprach bzw. textanalytischer Perspektive sind noch rar (vgl. Baron 2008; Honeycutt/Herring 2009; Meier i.Vorb. und die methodologische Diskussion in Markham/Baym (Hg.) 2009; abwesend hingegen bei Rowe/Wyss (Hg.) 2009). Fachliteratur gibt es lediglich zu Blogs, die hier jedoch nicht berücksichtigt werden. 
nikationsformen zu Leitkategorien für die konzeptionelle und analytische Gliederung des Feldes entwickelt, wie man an der Organisation mehrerer einschlägiger Publikationen erkennen kann. Gegen eine Fortführung dieser analytischen Trennung in der Web 2.0-Ära spricht zum einen das damit einhergehende Risiko eines latenten technologischen Determinismus, d.h. der impliziten oder expliziten Annahme, dass das Kommunikationsverhaltens durch die Kommunikationstechnologie bestimmt wird (Hutchby 2001). Indem man Sprachgebrauch im Internet prinzipiell nach der Kommunikationsform ausfächert, wird diese zum zentralen Kontextaspekt erhoben, was einen Vorrang technisch-medialer vor situativen und sozialen Kontextaspekten impliziert (vgl. kritisch dazu Androutsopoulos 2007b). Zum anderen nimmt die Unterscheidungskraft der Kommunikationsformen mit ihrer massenhaften Verbreitung ab: Wenn Emails gesamtgesellschaftlich allgegenwärtig sind, verliert die Kategorie „Sprache der Email“ ihre Bedeutung. ${ }^{5}$ Nicht zuletzt ist die analytische Orientierung an einzelnen Anwendungen nicht in der Lage, Kommunikationsprozesse zu erfassen, die sich quer durch verschiedene Plattformen erstrecken, beispielsweise die spezifische Koordination von Spielumgebung, Chat, Forum und Blogs bei Online-Gamern.

Der zweite Punkt betrifft die Dekontextualisierung von Sprache aus dem digitalen Kommunikationsprozess. Dies ist erst einmal eine legitime, gewissermaßen unvermeidliche methodische Konsequenz eines linguistischen Zugangs zu computervermittelter Kommunikation, sofern die Mikroanalyse sprachlicher Prozesse (u.a. das Verhältnis von Mündlichkeit/Schriftlichkeit, Aspekte von Sprachökonomie und -variation, Innovation und Wandel) eine Reduktion des semiotisch reichhaltigen Geschehens in Online-Umgebungen auf ihre (schrift-)sprachlichen Anteile voraussetzt. Damit geht oft auch ein auf die jeweilige Kommunikationsform reduziertes Kontext-Verständnis einher. In früheren Stadien internet-basierter Kommunikation wurde dieses Vorgehen zweifellos auch von den „schriftstarken“ Anwendungen begünstigt. Denn Chats, Newsgroups oder Emails sind semiotisch verhältnismäßig ,arme“ Umgebungen, in denen Schrift die zentrale, wenn nicht die ausschließliche semiotische Ressource darstellt. Allerdings stellen die gegenwärtigen, semiotisch reichhaltigen Web 2.0-Umgebungen dringende Fragen über den Anteil von Multimedialität und Multimodalität an der Erzeugung von kommunikativem Sinn und über das Zusammenspiel von Sprache mit anderen Zeichensystemen. ${ }^{6}$ Beispielsweise ist zu beobachten,

5 Vgl. bereits Schmitz (2002) und neuerdings auch Baron (2008). Damit geht die Vergänglichkeit digitaler Technologien im Medienwandel einher: Manche der früher gern untersuchten „interaktiven Dienste“ werden mit der Konvergenz des Web 2.0. allmählich obsolet. Newsgroups und Internet Relay Chat z.B. sind aus der Perspektive der heute heranwachsenden Generation von Internetnutzern bereits als veraltet zu bezeichnen (vgl. Thimm 2008, S. 333).

$6 \quad$ Ich unterscheide Multimedialität (Koexistenz und Kombination verschiedener Medien) und Multimodalität (Koexistenz und Kombination verschiedener semiotischer Modalitäten, Zeichensysteme in einem Text). 
dass dialogische Kommunikation in Vernetzungsplattformen eng mit Multimedia-Inhalten verzahnt ist, so dass z.B. ein eingebettetes Video als Auslöser für eine dialogische Sequenz auf einer Profilseite fungiert (siehe auch Abschnitt 6 unten). Gewiss lassen sich die Forschungsfragen der linguistischen Internetforschung auch an Web 2.0-Daten weiter verfolgen; durch eine voreilige Loslösung der linguistischen Komponente aus dem Bildschirmkontext und eine Reduktion desselben auf den Aspekt der Kommunikationsform wird man jedoch das Spezifische des Web 2.0 nicht in den Griff bekommen.

\section{Ein bildschirm-basierter Ansatz}

Statt einer einfachen Fortsetzung bisheriger analytischer Praktiken stellt uns Web 2.0 also vor die Aufgabe, einen neuen Rahmenansatz und ein neues Beschreibungsvokabular zu entwickeln, die auf gegenwärtige digitale Kommunikationsumgebungen abgestimmt sind und ihre Spezifika zu erfassen vermögen (vgl. auch Thimm 2008, S. 333).

In meinem Zugang werden computervermittelte Sprach- und Interaktionsprozesse in ein Spannungsfeld zwischen technisch-medialen Einschränkungen einerseits, sozialen und situativen Kommunikationsbedingungen andererseits platziert. Während die jeweils herangezogenen Kommunikationstechnologien einen Rahmen von Möglichkeiten und Einschränkungen abstecken, können sie einen spezifischen Sprachgebrauch nicht erzwingen oder determinieren; maßgeblich für diesen ist letztlich die individuelle bzw. gemeinschaftliche Aneignung dieser Technologien vor der Folie spezifischer sozialer und situativer Bedingungen. Hutchby bringt den Zusammenhang folgendermaßen zum Ausdruck:

We need to develop a framework that argues both that technologies for communication do indeed bring into existence - in the sense of enable and promote - new forms of participatory possibilities in human interaction, new categories of what might be called 'localized social identities', and that these new forms of interaction are at the same time the product of humans' active appropriation and configuration of the technology in pursuit of their own purposes. (Hutchby 2001, S. 7-8)

Daher ist die Untersuchung der sprachlichen Kennzeichen einzelner Kommunikationsformen zwar als erster deskriptiver Schritt notwendig, aber nicht ausreichend. Die Frage ist nicht nur, welche kommunikativen Möglichkeiten und Grenzen die Technologien des „Mitmachnetzes“ anbieten, sondern auch, wie vor der Folie dieser Bedingungen mit und in neuen Medien tatsächlich gehandelt wird.

Die Bezeichnung des Zugangs als „bildschirm-basiert“ verweist auf die Herkunft der untersuchten Daten und gleichzeitig auf den Kontrast zu nutzer-zentrierten Analysen ab, wie sie für Online-Forschung jenseits der 
Linguistik charakteristisch sind. Nutzer-zentrierte Ansätze legen den Schwerpunkt auf die Nutzer und ihre Aktivitäten vor dem Bildschirm, die mittels Interviews, Fragebögen, Fokusgruppen usw. untersucht werden. Ein bildschirm-basierter Ansatz konzentriert sich, ganz in der Tradition der linguistischen Internetforschung, auf Online-Texte bzw. -Aktivitäten. Vereinfacht gesagt sind die primären Analyseeinheiten nicht User, sondern Texte unterschiedlicher Größenordnung, die von einzelnen Webseiten über Diskussionsstränge bis hin zu gesamten Websites reichen. Der Ansatz schließt die ethnografische Beobachtung von Online-Prozessen sowie direkte Kontakte zu den Beteiligten mit ein (vgl. Androutsopoulos 2008).

Um der Komplexität von Web 2.0-Umgebungen gerecht zu werden, plädiere ich für eine Kombination von linguistischen bzw. semiotischen Analyseverfahren auf unterschiedlichen Strukturebenen in Verbindung mit einem dynamischen Verständnis von Interaktions- und Kontextualisierungsprozessen. Dabei werden Impulse aus der linguistischen Internetforschung, Sozio- und Diskurslinguistik, Medienlinguistik und Stilanalyse integriert (siehe Androutsopoulos 2007a, 2009; Coupland 2007; Herring 2004; Sandig 2006). Als oberste analytische Einheit ist die einzelne Webseite anzusehen. Auf Vernetzungsplattformen ist dies die einzelne Profilseite (einschließlich ihrer durch Tabellenreiter bzw. Navigationspunkte zu erreichenden Unterseiten), auf Mediaplattformen die einzelne Mediaseite (z.B. Video- oder Fotoseite) einschließlich des angeschlossenen Produzentenprofils und der Kommentare. Die Entscheidung für diese Analyseeinheit ist funktional und ethnografisch motiviert, d.h. dezidierte Profil- und Mediaseiten bilden jeweils funktionale Einheiten, an denen sich Online-Nutzer selbst orientieren. Am anderen Ende des analytischen Kontinuums liegen linguistische bzw. interaktionsanalytische Kategorien, beispielsweise der expressive Gebrauch von Orthografie oder Interpunktion, Ellipsen, sprachliche Höflichkeit usw. Das Ziel ist, den analytischen Fokus auf spezifische linguistisch-interaktionale Prozesse mit einem ganzheitlichen Verständnis der funktionalen Komplexität und Vielfalt von Web 2.0-Umgebungen zu verbinden.

In diesem Zusammenhang ist auch das Titel-Stichwort „Sprach-Gestalten" in seiner Ambiguität zu betrachten. Als Verbalkonstruktion verstanden - (durch) Sprache gestalten - bezieht es sich auf die kreativen Leistungen der Beteiligten, die sich in den gewählten digitalen Räumen ihre Identitäten und Beziehungen herbei schreiben; als Nominalkonstruktion - im Sinne von „Sprachgestalt" - verweist es auf die am Bildschirm sichtbaren semiotischen Konstrukte und Prozesse, die maßgeblich durch Sprache entstehen und sinnvoll gemacht werden. 


\section{Vier Leistungen von Sprache in Web 2.0-Umgebungen}

Vor diesem Hintergrund sollen nun zwei Kategorien-Sets vorgestellt werden, die in ihrem Zusammenspiel ein deskriptives Raster zur Erkundung von Web 2.0-Umgebungen ergeben. Ein dem anschließenden Fallbeispiel entnommenes Screenshot (Abb. 1) begleitet die Diskussion. Die erste Dimension ist die Unterscheidung von vier typischen Funktionen von Sprache in der Konstitution von Web 2.0-Umgebungen. Ich bezeichne sie mit den Stichworten Selbstdarstellung, Interaktion, Organisation und Spektakel.

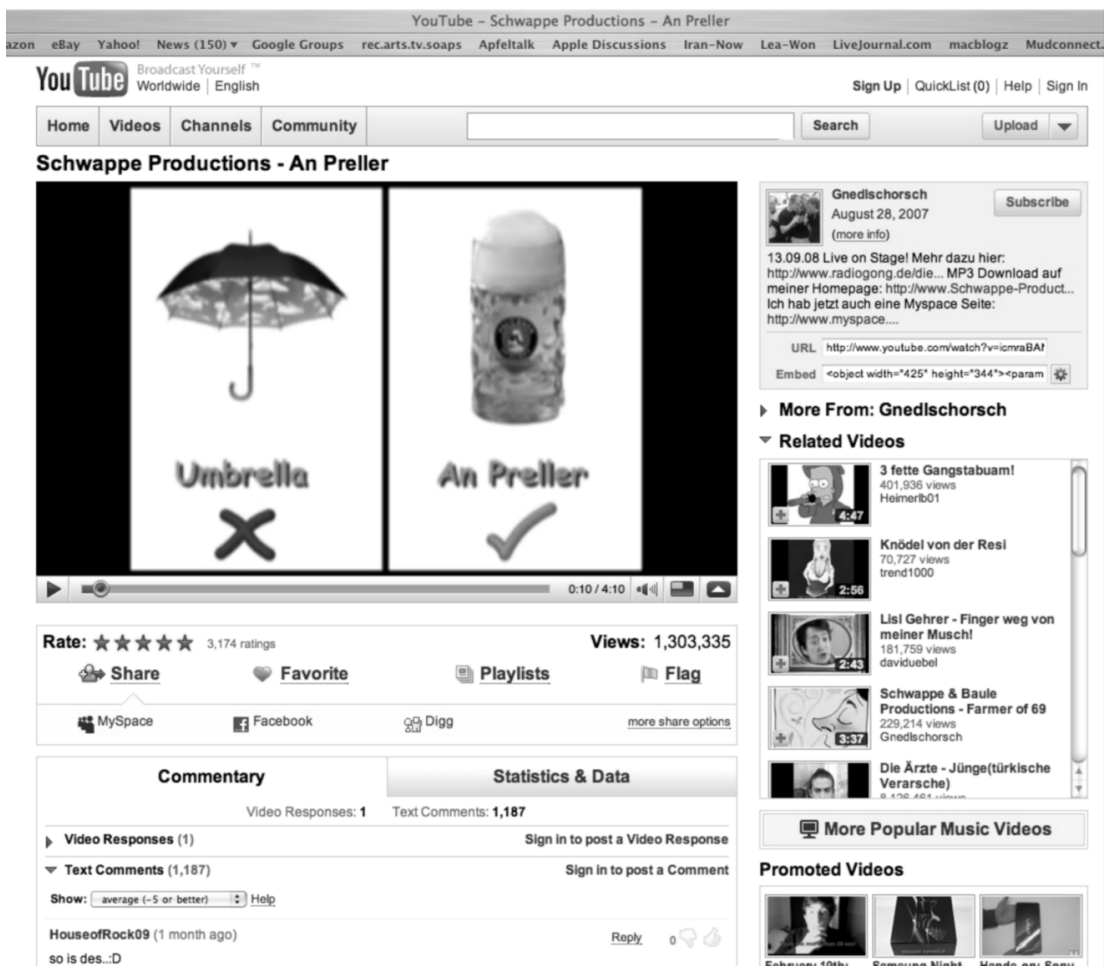

Abb. 1: Screenshot von „Schwappe Productions - An Preller“

Zunächst ist Sprache die wohl wichtigste (jedoch nicht die einzige) Ressource für die Selbstdarstellung der Nutzer auf Profilseiten. Sie wirkt dabei zusammen mit anderen semiotischen Möglichkeiten (Fotos, Weblinks, Hintergrundfarbe, Medieneinbettung usw.), die von allen gängigen Vernetzungsplattformen in unterschiedlichen Kombinationen angeboten werden. Heutige Profilseiten stehen in der Tradition der persönlichen Homepage, und Leitkategorien der früheren Homepageforschung sind auf sie anwendbar (vgl. Döring 2002; Chandler 1998; Stern 2008). Im Vergleich zu frü- 
heren Homepages sind heutige Profilseiten durch stärkere Serialisierung und Standardisierung einerseits, größere Spielräume für Individualisierung andererseits gekennzeichnet. Profilseiten fußen auf den generellen Voraussetzungen und Beschränkungen ihrer jeweiligen Vernetzungsplattform, die die äußere Form der Profile auf spezifische Weisen einschränken, indem sie bestimmte Modifikationsparameter erlauben oder ausschließen. Gleichzeitig verfügen die User über zahlreiche Optionen der Individualisierung, wozu auch ein leichter Umgang mit Multimedia gehört. Dadurch erweitert sich insbesondere das Repertoire der indirekten Hinweise auf Identität bzw. Persönlichkeit. Waren diese früher auf aufgelistete Weblinks beschränkt, können heute auch die eingebundenen Medianinhalte, die sichtbar aufgelisteten Freunde, die abonnierten Gruppen usw. die gleiche Funktion erfüllen. Auch die Verflechtung von Selbstdarstellung mit den Besucher-Rückmeldungen ist auf gegenwärtigen Profilseiten im Vergleich zu den früheren Gästebuchkommentaren enger geworden.

Die Interaktionsfunktion von Sprache - d.h. die Nutzung geschriebener Sprache zum interpersonalen Austausch - blickt ebenfalls auf eine längere Tradition der „getippten Gespräche“ (Storrer 2001) zurück, und frühere Forschungsergebnisse zur computervermittelten Interaktion scheinen auch für Web 2.0-Umgebungen in vielen Punkten noch gültig. Gleichzeitig werden die Möglichkeiten computervermittelter Interaktion neu kontextualisiert. Beispielsweise stellt die historisch recht neue, inzwischen weit verbreitete Kommentierung eines gegebenen Referenztextes (Blog-Beitrag, Video, Foto, Musik) spezifische Bedingungen der Kohärenzbildung dar. Anders als auf Foren bzw. Chats ist das Verhältnis zwischen den einzelnen Kommentaren weniger als Interaktion und mehr als Dialog mit dem Referenztext zu bezeichnen. Ähnliches gilt für die auf Vernetzungsplattformen zu beobachtenden Augenblicksdialoge, die sich anlässlich eines eingebetteten Videos bzw. einer Veränderung der Statuszeile entfalten.

In ihrer Organisationsfunktion trägt Sprache zusammen mit anderen semiotischen Mitteln (Farbe, Bilder, Piktogramme, Layout) dazu bei, die Bildschirmoberfläche als einen kohärenten virtuellen „Raum“ zu konstituieren, der Nutzerhandlungen ermöglicht und vorstrukturiert. Die zentrale Leistung sprachlicher Einheiten ist hier ihre Doppelfunktion als Überschriften und Hyperlink-Indikatoren, mittels derer Nutzer-Aktivitäten ausgeführt werden. Freilich ist diese Leistung konstitutiv für das WorldWideWeb überhaupt; spezifisch für Web 2.0 ist allenfalls ihre zunehmende Komplexität. Gegenwärtige Webseiten bestehen zu einem nicht geringen Anteil aus organisierender Sprache. Dazu zähle ich die verschiedenen Navigationsleisten, die überwiegend aus einfachen Nomina oder Verben, Nominal- bzw. Verbalphrasen bestehen. Auf jeder YouTube-Seite zum Beispiel (vgl. Abb. 1) findet man über dem Videofenster eine erste Navigationsleiste 
links (Startseite, Videos, Kanäle, Community), eine weitere oben rechts (Konto erstellen, Anmelden, Abonnements, Protokoll, Video bocbladen), eine Reihe von Optionen direkt unter dem Videofenster (Favorit, Weiterleiten, Playlists, Melden) usw. All diese lexikalischen Einheiten, deren Kohäsion zueinander und ihrer Umgebung multimodal strukturiert ist, stellen Hyperlinks dar, die die vorliegende Seite zu anderen Seiten bzw. Nutzeraktivitäten verknüpfen. Daran sieht man, dass die Organisationsdimension von Sprache von syntaktischer Reduktion charakterisiert ist und Textkohärenz innerhalb einer „visual syntax“ (van Leeuwen 2004, S. 17) hergestellt wird, der Typografie, Farbgebung und Layout angehören.

Dass die Organisationsleistung von Sprache in der linguistischen Internetforschung verhältnismäßig wenig Beachtung gefunden hat (vgl. aber Schmitz 2003 und in diesem Band), hängt sowohl mit dem Schwerpunkt auf interpersonaler dialogischer Netzkommunikation als auch mit einer allgemeinen Vernachlässigung von Multimodalität in der Linguistik zusammen (van Leeuwen 2004). Das enge funktionale Zusammenspiel mit anderen semiotischen Ressourcen bedeutet auch, dass die organisierende Sprache des Webs mit ausschließlich linguistischen Kategorien nur bedingt zugänglich ist. Zu den linguistisch zugänglichen - und spannenden - Fragen gehören beispielsweise die von globalen Web 2.0-Plattformen angebotenen Sprachoptionen und die stilistische Ausgestaltung der Linkbezeichnungen bzw. Navigationspunkte. Überprüft man beispielsweise, in welchen Sprachen das Videoportal YouTube verfügbar ist, so sieht man u.a. Nederlands oder Svenska, nicht aber Ukrainisch, Punjabi oder Arabisch. Daran schließt die Frage an, nach welchen Kriterien solche Sprachoptionen angeboten werden und wie sich die Relevanz der Sprecherzahlen zum wirtschaftlichem Gewicht einzelner Nationalsprachen verhält (vgl. auch Kelly-Holmes 2006).

Vor diesem Hintergrund heben sich Spektakel als wichtigste Innovation der Web 2.0-Ära ab. Als „Spektakel“ bezeichne ich Medientexte, die durch Nutzer auf Mediaplattformen hochgeladen und dort verfügbar gemacht werden. Es handelt sich in der Regel um multimodale bzw. -mediale Texte, die Sprache mit einschließen aber nicht auf diese beschränkt sind, typischerweise also Videos, Songs, Fotos. ${ }^{7}$ Die Metapher impliziert, dass diese Texte hauptsächlich als Unterhaltung wahrgenommen und ähnlich wie z.B. massenmediale Angebote konsumiert werden. Sie impliziert ferner, dass sie an eine Zuschauerschaft gerichtet sind und deren Reaktion suchen, die üblicherweise in Form von Kommentaren beigesteuert wird.

Die Bedeutung der Spektakel für die linguistische Untersuchung von Web 2.0 erschließt sich vor dem Hintergrund der Forschungslage in mehrfacher Hinsicht. Erstens eröffnet sich hier eine völlig neue Dimension von

Der Begriff umfasst zunächst Medientexte aller Art, selbst ohne Beteiligung von Sprache; in diesem Beitrag werden allerdings nur Videos berücksichtigt. 
„Sprache in neuen Medien“: War gesprochene Sprache im Netz bisher marginal, tritt sie nun in zahlreichen Kombinationen von Multimodalität und Gattung in Erscheinung. Spektakel sind bezüglich ihrer Gattungszugehörigkeit und stilistischen Beschaffenheit völlig offen, daher ist gesprochene Sprache in Spektakeln durch eine einfache Unterscheidung von konzeptioneller Mündlichkeit bzw. Schriftlichkeit noch nicht hinreichend beschrieben. Gleichzeitig sind Spektakel - wie unten noch im Detail auszuführen - mit der sie umgebenden digitalen Schriftlichkeit vielfach intertextuell verbunden.

Zweitens deutet die Spektakel-Metapher darauf hin, dass auf Mediaplattformen gestellte Nutzerproduktionen öffentliche Aufmerksamkeit jenseits des Massenmediensystems erlangen können. In meinen Beobachtungen und Fallanalysen von YouTube belege ich mehrere Beispiele für deutschsprachige Spektakel, die mehrere Millionen Zugriffe und tausende Kommentare erhalten, obwohl sie ihrer Ästhetik nach wohl keinen Zugang zum institutionellen Mediensystem gefunden hätten. Ein solcher Fall ist auch das in der Abbildung 1 gezeigte Video (siehe Abschnitt 7). Mit dem Kulturwissenschaftler John Fiske kann man hier sagen, dass auf Mediaplattformen außerinstitutionelle Primärtexte entstehen (Fiske 1987), die ganz ohne professionelle Produktion, „massentaugliche“ Ästhetik und sprachliche Standardisierung eine Massenaufmerksamkeit jenseits der Kontrollmechanismen der Medienindustrie nach sich ziehen (Androutsopoulos 2003). Dies ist soziolinguistisch insofern von Interesse, als Standardsprachlichkeit als Grundbedingung öffentlicher Kommunikation weiter aufgelockert und gleichzeitig auch die Sichtbarkeit von Nonstandardvarietäten potenziell gesteigert wird. Weitere linguistische Konsequenzen ergeben sich aus der Tatsache, dass Mediaplattformen Dreh- und Angelpunkt einer florierenden Kultur der multimedialen Aneignung und Verfremdung massenmedialer Materialien sind (vgl. hierzu Jenkins 2006). Viele Spektakel leben aus ihren intertextuellen Bezügen zur lokalen, nationalen oder auch globalen Populärkultur, und die Möglichkeit, Parodien von Massenkultur als Mittel sozialer oder politischer Kritik zu nutzen, gilt als Antrieb für die Popularität amateurhafter Videoproduktionen (siehe Jenkins 2006, S. 281 ff.).

Gleichzeitig sind Spektakel bezüglich ihrer Gattungszugehörigkeit wenig kontextualisiert. Ihnen fehlen die rahmenden Informationen, die in den konventionellen Massenmedien durch Sekundärtexte (in der Begrifflichkeit von Fiske 1987) wie Ankündigungen, Programmhinweise, Trailer, Rezensionen usw. geliefert werden. Bei Spektakeln hat man außer Titel, Tags und bestenfalls einigen Stichworten in der Produzentenbox (auf dem Bildschirm oben recht zu sehen) noch keine weitere rahmende Information. Dadurch entsteht eine Interpretationslücke, die in den Kommentaren aufgegriffen und teilweise auch gefüllt wird. 


\begin{tabular}{|l|l|l|l|}
\hline \multicolumn{1}{|c|}{ Dimension } & Hauptkennzeichen & \multicolumn{1}{|c|}{ Akteure } & \multicolumn{1}{c|}{$\begin{array}{c}\text { Typische } \\
\text { Umgebung }\end{array}$} \\
\hline Organisation & $\begin{array}{l}\text { Beitrag zum Web- } \\
\text { design }\end{array}$ & $\begin{array}{l}\text { Website-Administra- } \\
\text { toren bzw. -Designer }\end{array}$ & VP \& MP \\
\hline $\begin{array}{l}\text { Selbstdarstel- } \\
\text { lung }\end{array}$ & $\begin{array}{l}\text { Ressource für Profil- } \\
\text { seiten }\end{array}$ & $\begin{array}{l}\text { Individuelle Nutzer } \\
\text { (Profilseitenbesitzer) }\end{array}$ & VP \\
\hline Spektakel & $\begin{array}{l}\text { Teil des von Nutzern } \\
\text { hochgeladenen Multi- } \\
\text { media-Materials }\end{array}$ & Nutzer & $\begin{array}{l}\text { MP (und ein- } \\
\text { gebettet in } \\
\text { VP) }\end{array}$ \\
\hline Interaktion & $\begin{array}{l}\text { Interpersonale Kom- } \\
\text { munikation zwischen } \\
\text { Nutzern, Kommentie- } \\
\text { rung von Spektakeln } \\
\text { und anderen Referenz- } \\
\text { texten }\end{array}$ & $\begin{array}{l}\text { Mehrfachautoren- } \\
\text { schaft }\end{array}$ & VP \& MP \\
\hline
\end{tabular}

Tab. 1: Vier Dimensionen von Sprache in Web 2.0-Umgebungen (VP: Vernetzungsplattformen; MP: Mediaplattformen)

Die vier Dimensionen von Sprache in Web 2.0-Umgebungen, deren Hauptkennzeichen in Tabelle 1 zusammengefasst werden, treten in der Empirie stets in Kombinationen auf. So besteht eine enge Verbindung zwischen Selbstdarstellung und Interaktion, indem Elemente der persönlichen Selbstdarstellung und Besucherkommentare nebeneinander erscheinen und Bezug aufeinander nehmen (beispielsweise wenn auf facebook die Veränderung der Statuszeile eine Kommentarsequenz veranlasst). Die Dimensionen der Selbstdarstellung und des Spektakels treten in einen Dialog dadurch, dass auf Profilseiten eingebettete Videos, Musikstücke oder Fotos als Mittel der Selbstdarstellung herangezogen und interaktiv kommentiert werden. Ein Zusammenspiel von Spektakel und Interaktion ist für alle Mediaplattformen konstitutiv, indem hochgeladenen Inhalte von Zuschauern kommentiert und bewertet werden. Diese Wechselwirkungen und Querverbindungen haben analytisch zur Konsequenz, dass man stets Relationen zwischen den einzelnen Dimensionen und deren Erscheinungsformen unter die Lupe nimmt.

\section{Diskursprozesse in Web 2.0-Umgebungen}

In einem zweiten Schritt unterscheide ich für Web 2.0-Umgebungen typische Diskursprozesse und belege diese mit den Bezeichnungen „Multimodalität", „Intertextualität“ und „Heteroglossie“. Dieses zweite Kategorien-Set fokussiert auf die Sprach- bzw. Textanalyse. Gefragt wird nicht nach den Leistungen von Sprache in der Konstituierung von Online-Kom- 
munikation, sondern nach Eigenschaften von konkreten Online-Texten und -Interaktionen. Dabei besteht keine einfache Korrespondenz zwischen den beiden Kategoriensets. Beispielsweise tritt Multimodalität in allen vier Funktionsdimensionen in Erscheinung, und ein und dieselbe Funktionsdimension kann multimodale, intertextuelle und heteroglossische Züge aufweisen. Auch hier geht es mir um Facetten der drei Prozesse, um ihre vielfältigen Erscheinungsformen und ihr Verhältnis zu massenmedialen bzw. netzspezifischen Diskurstraditionen. Die Frage ist also nicht: „Was sind diese Prozesse?“, sondern: „In welchem Sinne kann man von ihnen sprechen, und wie treten sie in Web 2.0 in Erscheinung?"

Die Dimension der Multimodalität lenkt unsere Aufmerksamkeit auf die Gesamtheit der semiotischen Ressourcen, die auf einer Webseite in spezifischen Kombinationen miteinander auftreten und zur Konstitution textueller Einheiten beitragen. Neben den in allen visuellen Medien üblichen Sprache/Bild-Kombinationen umfassen Web 2.0-Seiten weitere Verbindungen aus Text, Ton und bewegtem Bild. Ihre Fülle und Ubiquität legen nahe, dass der veränderte Umgang mit Multimedia und Multimodalität ein zentrales Kennzeichen von Web 2.0-Umgebungen darstellt. Wie multimodale Kompositionen zu analysieren sind, dafür stehen verschiedene Ansätze zur Verfügung, auf die hier nicht weiter eingegangen werden kann. ${ }^{8}$ Ein Desideratum ist der Vergleich zwischen Plattformen hinsichtlich ihrer Palette an multimodalen Optionen sowie zwischen Nutzungsstilen hinsichtlich der tatsächlichen Umsetzung dieser Optionen. Beispielsweise unterscheiden sich gängige Vernetzungsplattformen darin, welche multimodalen Ressourcen zugelassen oder eingeschränkt werden. So lässt MySpace die Manipulation von Hintergrund-, Box- und Schriftfarben zu und erlaubt die freie Platzierung von Textboxen sowie die Einbettung von Musik, während all diese Optionen der Selbstdarstellung auf anderen Plattformen nicht verfügbar sind (vgl. boyd 2008). Eine weitere Frage ist nach den Wechselwirkungen von Multimodalität und verbaler Online-Interaktion. Die Einbettung multimodaler Materialien in einen Interaktionsbeitrag bzw. -strang gehört genauso dazu wie die Frage, wie sich die Orientierung der Kommentare auf ein Foto oder Video deiktisch niederschlägt.

Auch im Bereich der Intertextualität - der Bezugnahme von Texten auf andere Texte - lassen sich „ältere“ und „neuere“ Erscheinungsformen unterscheiden. Bestimmte Spielarten intertextueller Beziehungen auf Web 2.0Seiten sind genauso in anderen Medien vorzufinden und greifen auf rhetorische Traditionen zurück. Dies gilt insbesondere für die „enge“ intertextuelle Bezugnahme auf spezifische Prätexte, die unverändert oder verändert (als Zitate, Anspielungen usw.) in den Referenztext eingehen und dort typischerweise an funktional exponierten Stellen wie Titeln und Überschriften erscheinen. Darüber hinaus sind in Vernetzungs- und Mediaplattformen

$8 \quad$ Z.B. Kress/van Leeuwen (2001); Stöckl (2004); Schmitz (2003). 
auch innovativere intertextuelle Spielarten zu beobachten. Ein Beispiel ist die zitathafte Verwendung von Hyperlinks und eingebetteten Inhalten. So kann auf Vernetzungsplattformen beobachtet werden, dass Kommentare auf einer Profilseite auch Photos, Videos oder Hyperlinks enthalten, welche den sprachlich ausformulierten Beitrag nicht (nur) ergänzen, sondern vielmehr ersetzen: Man antwortet sozusagen durch ein Bild, ein Video oder einen Link, so dass diese Beitrags-, bisweilen auch Sprechhandlungscharakter gewinnen.

Besondere Beachtung verdient das Geflecht von Textbeziehungen innerhalb der bzw. rund um Spektakel. Auf der Videoplattform YouTube manifestiert sich Intertextualität zunächst einmal als Beziehung zwischen einem Spektakel und den (massenmedialen) Quellen, die dieses zitiert, verwandelt oder parodiert. Intertextuelle Bezüge prägen auch das Verhältnis zwischen Spektakeln und den umliegenden Seitenelementen. Während Verbindungen thematischer oder gattungsspezifischer Art zwischen einem Spektakel und den in der Seitenleiste gelisteten ,ähnlichen Videos“ durch die Plattform-Datenbank geknüpft werden, sind Verbindungen zwischen Spektakel und einer „Video-Antwort“ das Ergebnis gezielter Nutzeraktivität, um in einen filmischen Dialog mit einem Referenzvideo zu treten. Nicht alle Videos erhalten Videoantworten, sie können aber weit mehr als nur eine erhalten, so dass die Videoantwort auch als Indikator von $\mathrm{Zu}$ schauer-Engagement verstanden werden kann. Ein weiterer intertextueller Verbund entsteht zwischen Spektakel und anschließender Kommentierung. Dabei dienen Kommentare nicht einfach der (sachlichen oder affektiven) Bewertung, sondern leisten weitere rhetorische und kontextualisierende Funktionen: Ähnlich wie Zuschauerkommentare können sie Fragmente des Spektakels aufnehmen und wiederholen, Aspekte seiner lokalen Rezeption und Zirkulation aufgreifen. Damit können sie auch Kontext- und Hintergrundinformation liefern, die den meisten amateurhaft produzierten Spektakeln fehlen. Diese Bemerkungen zeigen, dass ,Intertextualität sowie das weitere damit verbundene Begriffsfeld als deskriptive und interpretative Schlüsselkategorien für die Untersuchung von Web 2.0-Umgebungen geeignet ist.

Das Konzept der ,Heteroglossie‘ bezieht sich auf das Verhältnis zwischen Sprachen bzw. Sprechweisen mit unterschiedlichen sozialen bzw. ideologischen Perspektiven innerhalb einer Äußerung oder eines Textes. Der Begriff wurde von der Sprach- und Diskurstheorie von Mikhail Bakhtin (Bakhtin 1981) in die Literatur- und Sprachwissenschaft übernommen und ist bereits auf die Untersuchung von Mediendiskursen übertragen worden (siehe z.B. Busch 2004). Gängige Definitionen von Heteroglossie (etwa bei Bailey 2007, S. 257) heben zum einen auf die Koexistenz von verschiedenen Zeichen oder „Stimmen“ innerhalb einer Äußerung bzw. eines Textes $\mathrm{ab}$, zum anderen auch auf die Spannung und Konfliktrelation zwischen die- 
sen Zeichen aufgrund ihrer gesellschaftlichen und historischen Assoziationen. ${ }^{9}$ Damit geht Heteroglossie an einem entscheidenden Punkt über den Begriff der sprachlichen Variation hinaus, der in der linguistischen Internetforschung Einsatz findet (vgl. Beiträge in Androutsopoulos 2006). Während Variation und Variationsanalyse quantitative Verhältnisse zwischen verschiedenen (Bündeln von) Formvarianten innerhalb einer Einzelsprache erfassen, fängt Heteroglossie auch ein weiteres Kennzeichen von Sprache im Internet auf: die immer wieder hervorgehobene Koexistenz, Mischung oder auch Kontrastierung verschiedener Sprachen, Varietäten oder Sprachstile, gegebenenfalls auch in multimodalen Zusammenhängen. ${ }^{10}$

Analytisch ist Heteroglossie ein relationales Konzept, d.h. man identifiziert nicht einzelne sprachliche Varietäten oder Stilmerkmale, sondern Relationen zwischen Elementen die in einem Diskursausschnitt koexistieren. In Vernetzungs- und Mediaplattformen sind solche Relationen an mehreren Erscheinungsstellen potenziell zu finden. Durch Konvergenz (siehe Abschnitt 2) entsteht in Web 2.0-Umgebungen eine Vielzahl von „,kleinen“ Gelegenheiten der Koexistenz von unterschiedlichen Sprech- und Schreibweisen, die man mit dem Konzept der Heteroglossie auf das Verhältnis zwischen semiotischer und soziokultureller Differenz überprüfen kann. Ein Beispiel ist die Koexistenz von professionell und nutzerseitig erstellten Inhalten, die durch ihren Stil die unterschiedliche Rolle und Perspektive, der sie entstammen, kontextualisieren. Für eine weitere Bestimmung kann man zunächst auf die vier Funktionsdimensionen von Sprache in Web 2.0 zurückgreifen:

- Zwischen Organisation und den anderen drei Funktionsdimensionen können heteroglossische Kontraste dadurch entstehen, dass die Sprache der Spektakel bzw. interaktiven Beiträge mit der Sprache des Organisationsrahmens nicht übereinstimmt. Für Sprecher einer in den Einstellungsoptionen der Plattform nicht enthaltenen Sprache ist der Kontrast zwischen Sprache der Inhalte und Sprache der Seitenbedienung allgegenwärtig.

- Zwischen Spektakel und Interaktion entstehen heteroglossische Spannungen dadurch, dass sich Sprache im Spektakel von den konversationellen Stilen der Kommentareinträge unterscheidet, wobei solche Unterschiede auch selbst Gegenstand der Kommentierung werden können.

9 Bakhtin beschreibt Heteroglossie u.a. als „,co-existence of socio-ideological contradictions between the present and the past, between differing epochs of the past, between different socio-ideological groups in the present, between tendencies, schools, circles and so forth" (Bakhtin 1981, S. 291). Duranti schreibt dazu: ,the speech of any one person is filled by many different voices or linguistically constructed personae" (Duranti 1997, S. 75 f.).

10 Vgl. u.a. Runkehl/Schlobinski/Siever (1997); Georgakopoulou (2003); neuerdings auch Tsiplakou (2009) und Tagliamonte/Denis (2008). Letztere sehen in der „consistent juxtaposition of ,forms of a different feather" ein ,quintessential characteristic of IM discourse“ (Tagliamonte/Denis 2008, S. 26). 
- Innerhalb der Funktionsdimensionen der Selbstdarstellung und Interaktion können heteroglossische Relationen dadurch entstehen, dass die Beteiligten die sozialen Assoziationen verschiedener Sprachstile mehr oder weniger bewusst nutzen, um unterschiedliche Standpunkte, Beziehungsstrategien usw. zu kontextualisieren (vgl. Androutsopoulos 2007a).

\section{Ein Fallbeispiel: Varietätendynamik auf YouTube}

Abschließend möchte ich an einem Fallbeispiel aufzeigen, wie auf der Grundlage der vorgestellten Leitkategorien weiterführende Fragestellungen aufbauen können. Das Beispiel bezieht sich auf Formen und Funktionen sprachlicher Varietäten in der computervermittelten Kommunikation und greift damit eine Frage auf, die in der Forschung bereits beachtet worden ist, sich aufgrund der erläuterten Bedingungen der gegenwärtigen WebUmgebungen jedoch erneut und unter veränderten Vorzeichen stellt. Ging es beispielsweise in der Chat-Kommunikation um die schriftliche Repräsentation von Regionaldialekten und ihren strategischen Einsatz in OnlineInteraktionen (vgl. z.B. Siebenhaar 2006), so stellen sich mit Blick auf Mediaplattformen auch die Fragen, welche Möglichkeiten die „Medienproduktion von unten " für die Repräsentation lokaler Sprechweisen eröffnet, ob außerinstitutionell produzierte Spektakel neue Freiräume für Dialekte anbieten und wie die soziale Bedeutung von Varietäten in Spektakeln und ihren Kommentaren ausgehandelt wird. ${ }^{11}$

Als Beispiel für diese Fragestellungen sei das Bairische herangezogen. Empirischer Ausgangspunkt für die Frage, wie und wozu bairische Dialekte in Spektakeln verwendet werden, sind die Schlagworte („Tags"), mit denen Videos auf Mediaplattformen versehen werden. Videos mit dem Attribut „bairisch“ (bzw. auch bayrisch, boarisch) lassen einen nicht näher definierten Bezug zum Bairischen erwarten, dessen genauere Beschaffenheit analytisch festzustellen ist. Eine erste Sichtung des YouTube-Bestandes an derart getaggten Videos ${ }^{12}$ führt zur Unterscheidung von drei Kategorien, die sich von ihrer Herkunft und Produktionsweise her unterscheiden: a) Auszüge aus Filmen, Serien oder sonstigen bereits veröffentlichen Medieninhalten, b) Eigenproduktionen (z.B. selbst aufgenommene Szenen in einem Bierzelt), c) Modifizierungen massenmedialen Materials (z.B. „PulpFiction auf Bayrisch").

11 Die nachfolgende Diskussion greift auf Überlegungen zurück, die gemeinsam mit Horst Simon (King's College London) entwickelt wurden.

12 Am 12. Juni 2009 fand man auf YouTube 912 mit bayrisch, 206 mit bairisch und 262 mit boarisch getaggte Videos. Dabei bezieht sich bairisch speziell auf die bairische Dialektgruppe, bayrisch auf die Region Bayern (faktisch aber auch auf die bairischen Dialekte) und boarisch ist eine dialektal markierte Variante. Das Verhältnis der Tags zum Inhalt der Videos und deren regionalen und regionalsprachlichen Orientierung müsste freilich erst einmal genauer untersucht werden. 
Alle diese Videos sind grundsätzlich multimodal, sofern sie gesprochene Sprache, Ton und bewegtes Bild (und möglicherweise auch Untertitel) beinhalten. Wir können fragen, wie die jeweiligen multimodalen Kombinationen zur Konstitution eines „bairischen“ Bezugs beitragen. Zweitens können „bairische“ Videos verschiedene intertextuelle Relationen aufweisen. Dies ist vor allem bei jenen Videos der Fall, die das mediale Modifizierungspotenzial des Web 2.0 durch die Neuvertonung, -bebilderung oder -synchronisierung medialer Materialien stärker ausnützen. Solche Videos haben auch ein heteroglossisches Potenzial, das sich in ihrer Relation zu Quelle bzw. zu den umgebenden Textelementen zeigt. Beispielsweise können Dialekt/ Hochdeutsch-Kontraste humoristisch oder parodistisch aufgebaut werden.

Solche heteroglossischen Relationen scheinen in der Gruppe der modifizierten „bairischen“ Videos, die mit der Verfremdung massenmedialen Materials arbeitet, am deutlichsten ausgeprägt. Ein Beispiel für dieses Verfahren ist das Amateurvideo "An Preller“ (Abb. 1), das den US-amerikanischen Popsong „Umbrella“ als Grundlage benutzt, wobei die Originalmelodie von einem neuen Liedtext überlagert und mit einem neuen Video versehen wird. ${ }^{13}$ Es handelt sich gewissermaßen um eine „bebilderte Kontrafaktur", sofern die Melodie behalten, der Text aber umgedichtet und der Song durch eine Sequenz statischer Bilder ,umbebildert" wird. ${ }^{14}$

Textbeispiel 1: Songtext „An Preller“, erste Strophe und Refrain (0:33-1:13), Verschriftung nach den Untertiteln

1. Am Wochenend in Bayern / gengan die Leid gern feiern

2. I mach des a recht gern / Noch ist der Absturz fern

3. Aber dann kaffst da a Mass / Und scho steigt der Spaß

4. Nach Nummer 8 jedoch / Hat der Spaß boid a Loch

5. I hob / scho wieder an Rausch in der Fotzn / Hearst des is doch echt zum Kotzen

6. Koaner versteht mi wei i so lall / Zefix bin I scho wieder prall

7. Draußt werds scho langsam wieder heller / Aber mi drabts nur oibe schneller

8. Wei i hob scho wieder an so an Preller! / i hob scho wieder an so an Preller!

Auf Basis des vorgestellten Ansatzes lässt sich „An Preller“ auf seine multimodale Konstitution, intertextuelle Verflechtung sowie heteroglossische Zusammensetzung untersuchen. Im Folgenden werden diese drei Schritte als Hintergrund gesetzt und die Aufmerksamkeit auf die Stilisierung des bairischen Dialekts im Video gerichtet. Heuristische Leitfragen der Analyse sind:

13 Das Video ist unter http://www.youtube.com/watch?v=icmraBAN4ZE abrufbar, eine Lektüre begleitende Rezeption wird empfohlen. Hintergrundinformation zum Original bietet http://en. wikipedia.org/wiki/Umbrella_(song). Die 2007 nur wenige Monate nach dem Original publizierte „bairische Fassung“ weist zum Erhebungsstichtag 1.362.584 Aufrufe, 1.235 Kommentare und zwei Videoantworten auf (Stand: 12.06.2009).

14 Das Stichwort „Kontrafaktur“ verdanke ich Jana Tereick. 
- Welche bairischen Dialektmerkmale liegen vor?

- Wie wird Dialekt im Video behandelt, wie ist sein Einsatz durch Gattung, Handlungsstruktur und Bildkomponente motiviert?

- Wie gehen die Kommentare mit Dialekt als Teil des Spektakels bzw. als eigene stilistische Ressource um?

- Wie wird Dialekt im Spektakel bzw. in den Kommentaren mit sozialen Kategorien gekoppelt?

- Allgemein, wie gewinnt der Dialektgebrauch seinen Sinn unter den Rahmenbedingungen der Videoplattform?

Meine These lautet, dass die Bedeutung des Dialektgebrauchs in „An Preller“ und anderen „bairischen“ YouTube-Spektakeln nicht allein aus der Dialektalitätsstufe und nicht nur aus der sprachlichen Dimension dieser Videos zu erschließen ist. Vielmehr entsteht sie in der Zusammenwirkung aller semiotischen Dimensionen des Spektakels sowie in dem Dialog zwischen Spektakel und Kommentaren.

In den bisher gesichteten „bairischen“ YouTube-Videos sind die Stufe und der Umfang des Dialektgebrauchs nicht von vornherein abgegrenzt. Realistischer, konversationeller Dialekt kommt genauso vor wie ein stilisierter Dialekt mit einzelnen, salienten Merkmalen vor der Folie einer regionalen Standardsprache (vgl. Birkner/Gilles 2008). Im Beispiel „An Preller" findet ein „flaches“, urbanes Bairisch Verwendung. Dennoch weist die (männliche) singende Stimme ausreichende und konsistente Unterschiede zum Standarddeutschen auf - in der Lautung, im Wortschatz und teilweise auch der Syntax - um als Dialektstimme eingestuft zu werden. Typische Merkmale des Bairischen finden sich im Vokalismus, den Pronomina sowie im Dialektwortschatz, das durch seine Platzierung im Titel und Refrain eine wichtige Rolle bei der Identifizierung des Textes als „dialektal“ spielt. Ein Dialektausdruck (an Preller, ein Rausch') dient als Dreh- und Angelpunkt der Parallelisierung zwischen den beiden Fassungen.

Die Analyse des Dialekts im gesprochenen Songtext ist zu ergänzen durch die sprachliche wie auch bildliche Kontextualisierung von Dialekt im Video. Auffallend ist zunächst, dass der Songtext dialektal untertitelt wird (vgl. Textbeispiel 1), was die Präsenz von Dialektalität im Video weiter verstärkt. Andere Aspekte von Schriftsprache im Video verstärken seinen heteroglossischen Charakter. Standarddeutsch ist in den Endtiteln sowie in mehreren Bildern (als eingebettete Legende) zu lesen, stellenweise sind auch Englisch (do not disturb) sowie „bairisches Englisch“ (Pardy ends, mit Abbildung der Lenisierung des alveolaren Plosivs) zu sehen. Damit wird die im Video dominante Dialektstimme durch schriftliche Spuren anderer Sprachen bzw. Varietäten ergänzt.

Daraus geht hervor, dass die Leistung von Dialekt als Lokalitätsmarker ohne eingehende Beachtung des Bildtextes nicht vollständig einzuschätzen ist. Daher ist die linguistische durch eine multimodale sowie intertextuelle 
Analyse zu ergänzen, bei denen die Beschaffenheit des Bildtextes, SpracheBild-Passungen sowie der Beitrag des Bildlichen zur Konstituierung lokaler Bezüge und Identitäten im Vordergrund stehen. Leitfragen sind hier: Welches semiotische Material wird im Bildtext des Spektakels als Folie herangezogen und kombiniert? Wie ist das Verhältnis zwischen der dialektalen Stimme und dem bewegten Bild strukturiert?

Grundsätzlich haben wir es bei „An Preller“ mit einer Bricolage (Chandler 1998) zu tun, bei der Bilder aus verschiedenen Referenzbereichen neu zusammengesetzt werden. Sequenziell vorgehend ist der Beginn des Videos mit zahlreichen lokalen Emblemen durchsetzt. Noch vor dem Beginn des Songtextes (0:06) sieht man eine Gegenüberstellung eines Regenschirmes („Umbrella“) und der bairischen Maß, die das Titel gebende Wortspiel visualisiert (siehe Abb. 1). Bayerische Flagge und Landkarte sowie die Paulaner$\mathrm{Maß}$ leisten in den ersten 60 Sekunden eine lokale Einrahmung des Spektakels und begleiten dabei die sprachliche Konstitution des lokalen Kontextes (vgl. Textbeispiel 1, Zeilen 1-2). Anschließend werden aus Webforen bekannte Smiley-Grafiken mit trinkenden und feiernden Figuren gezeigt, wodurch die Zeilen 2-4 bebildert werden. Quer durch das gesamte Video werden Regionalembleme mit Bildern aus der deutschen Populärkultur ${ }^{15}$ und der Internetkultur zusammengemischt und sequenziell dem Songtext angepasst. Die Bilder sind dabei ikonisch oder metonymisch zum Gesagten platziert, sie werden dadurch desambiguiert und tragen zur Kohärenz des multimodalen Gesamttextes bei. Gleichzeitig sind sie im Sinne einer visuellen Intertextualität interpretierbar, d.h. tragen durch ihre Ästhetik und Herkunftsdomänen zur (komplexeren) Interpretation und kulturellen Positionierung des Gesamttextes bei. Wichtig ist, dass hier keine ästhetische Einheitlichkeit, sondern eine Kontrastierung von lokalem, nationalem, globalem Material entsteht.

Die Analyse des Spektakels ist im Sinne der aufgestellten Programmatik durch die der Kommentare zu ergänzen, die auf ihre Sprechhandlungen, ihre intertextuellen Bezüge zum Spektakel bzw. untereinander sowie ihre stilistischen Selektionen untersucht werden. Gefragt wird, wie die Kommentare den lokalen Bezug und die lokale Rezeption des Videos thematisieren; ob und wie sie den Dialektgebrauch kommentieren; in welcher Varietät bzw. Stillage sie verfasst sind, und ob dadurch - in Abhängigkeit von Referenz und Sprechhandlung - eine Konvergenz zum bzw. Divergenz vom Sprechstil des Videos erzielt wird.

So fällt bei der Analyse eines Samples ${ }^{16}$ auf, dass viele Kommentare zu "An Preller" eine Beziehung zum Bairischen bzw. zu Bayern durch propositionalen Gehalt, intertextuelle Referenzen oder den eigenen Dialekt-

\footnotetext{
15 Wieder erkennbare Bilder sind u.a. der Altkanzler Kohl (2:29) und das Sandmännchen (3:44).

16 Gesichtet wurde ein Sample von 500 Kommentaren bzw. ca. 40\% der Gesamtanzahl zum Zeitpunkt der Erhebung.
} 
gebrauch herstellen. Insbesondere positive Bewertungen und affirmative Kommentare sind öfters im Dialekt und können dadurch als Fälle von Konvergenz angesehen werden. Das Verhältnis von „An Preller“ zum Originalsong wird gelegentlich als antagonistisch thematisiert (ca. 15 Fälle in meinem Sample, eine Auswahl sieht man in Textbeispiel 2), d.h. „An Preller" wird als eine Art symbolischer Widerstand zum Original, das im Sommer 2007 intensiv in den Massenmedien gespielt wurde, gefeiert und explizit als Parodie desselben klassifiziert.

Textbeispiel 2: Kommentare zu An Preller mit Bezug auf das Original

- so sebr wie ich das original hasse, liebe ich diese version

- Also des is die viel bessere Version von Umbrella $=D$

- von wegen parodie das hier is das original ;) umbrella is eh en scheiß lied aber das bier wird bald kult sein

Textbeispiel 3: Kommentare zu An Preller mit Bezug auf seinen lokalen Umlauf

- seit tagen singen wir den song an jeder party!

- in unsana niederbayerischen schui kursiert des scho lang wieder auf de handys...

- will ich im Radio bören!

Darüber hinaus lassen die Kommentare Aspekte der lokalen Nutzung und Popularität des Videos erkennen (Textbeispiel 3). Beispielsweise impliziert die gestellte Frage, ob man das Lied herunterladen könne, eine Nutzungsabsicht in Situationen jenseits des Rechners und des Internet. Es finden sich Hinweise, dass der Song auf Partys gespielt werden sollte bzw. gespielt wurde, wobei diese Nutzungsberichte teilweise selbst im Dialekt verfasst sind. Andere Kommentare setzen „An Preller“ pro- oder retrospektiv in Kontext des Oktoberfestes 2007, etwa mit der Prognose Wiesnhit 2007! oder dem Hinweis, dass der Song tatsächlich in den Partyzelten gespielt wurde. An diesen Beispielen erkennt man, wie bereits in der theoretischen Diskussion angedeutet, die vielfältigen Diskursfunktionen der Kommentare und ihre Kontextualisierungsleistung, die sich in drei Bereichen verdichtet: Metasprachliche Dialektthematisierung, antagonistischer Vergleich zum Original und Hinweise auf die lokale Rezeption und Zirkulation des Songs.

\section{Schlussfolgerung und Ausblick}

Die Analyse hat den Dialektgebrauch im YouTube-Video „An Preller“ in einem mehrfachen Zusammenhang verortet: in der Zusammenwirkung der verschiedenen Medien und Modalitäten, dem Verhältnis zwischen Spektakel und anschließenden kommentierenden Diskurs sowie dem Verhältnis 
zur Konstruktion lokaler Identitäten. Zentral für die Sinnstiftung des Dialekts ist in diesem Beispiel die multimodale Inszenierung lokaler Stereotype vor der Folie globaler Populärkultur. ${ }^{17}$ „An Preller“ präsentiert sich als Stilisierung lokaler Kultur und Lebenswelt, und der Dialekt wird als „natürliche" Stimme dieser Lebenswelt platziert, was in den Kommentaren eher bestätigt als bestritten wird. Gleichzeitig ist der Dialekt Teil einer von Kontrasten geprägten, heteroglossischen Gegenüberstellung zum Original: Der weiblichen, professionellen, leidenschaftlichen Stimme von Rihanna steht hier eine männliche, untrainierte, apathisch klingende Stimme entgegen, der Romantik und aufwändigen Produktion des Originaltextes wird eine amateurhafte Trash-Ästhetik gegenübergestellt. Dies führt nicht zu einer negativen Rezeption des „bairischen“ Spektakels in den Kommentaren, sondern im Gegenteil zu einer begeisterten Aufnahme. „En Preller“ - wie auch andere als „bairisch“ verschlagwortete Videos auf YouTube - wird wohl als eine lokale Antwort auf globale Popkultur interpretiert und gefeiert, und die Bedeutung des Dialektgebrauchs ist auch in diesen Kontext zu stellen. In anderen Worten erschöpft sich der soziale Sinn des Dialekts nicht auf eine tradierte Verbindung zwischen Sprache und lokaler Identität, sondern diese Verbindung wird als Lokalstereotyp in einem Dialog zwischen globaler und lokaler Kultur evoziert und durch Verfahren der Multimodalität, Intertextualität und Heteroglossie herausgearbeitet.

Der Beitrag hat diese drei Konzepte als Grundlage eines deskriptiven Zugangs zu Sprache in Web 2.0-Umgebungen vorgeschlagen. Angesichts der semiotischen und medialen Reichhaltigkeit, die Web 2.0-Umgebungen kennzeichnet, werden inklusive und relationale sprach- und diskursanalytische Zugänge benötigt, die „cross-modally“ (van Leeuwen 2004, S. 15), d.h. an Sprache, Bildern und anderen Modalitäten angewandt werden können und Relationen zwischen Modalitäten, Texten und Codes in den Vordergrund rücken. Bei angemessener empirischer Weiterentwicklung können diese Kategorien einen ganzheitlichen Zugang zu gegenwärtigen Web-Umgebungen ermöglichen, der die in der linguistischen Internetforschung bisher verfolgten Fragestellungen nicht ausschließt oder ersetzt, sondern integriert und kontextualisiert.

\section{Literatur}

Androutsopoulos, Jannis (2003): HipHop und Sprache: Vertikale Intertextualität und die drei Sphären der Popkultur. In: Androutsopoulos, Jannis (Hg.): HipHop: globale Kultur - lokale Praktiken. (= Cultural Studies 3). Bielefeld, S. 111-136.

Androutsopoulos, Jannis (2006): Introduction: Sociolinguistics and computer-mediated communication. In: Journal of Sociolinguistics 10, 4, S. 419-438.

17 Dieser Zusammenhang wird in Androutsopoulos (ersch.demn.) vertieft. 
Androutsopoulos, Jannis (2007a): Style online: Doing hip-hop on the German-speaking Web. In: Auer, Peter (Hg.): Style and social identities. (= Language, Power and Social Process 18). Berlin/New York.

Androutsopoulos, Jannis (2007b): Neue Medien - neue Schriftlichkeit? In: Mitteilungen des Deutschen Germanistenverbandes 1, S. 72-97.

Androutsopoulos, Jannis (2008): Potentials and limitations of discourse-centered online ethnography. In: Language@Internet 5, article 8. Internet: www.languageatinternet. de/articles/2008 (Stand: November 2009).

Androutsopoulos, Jannis (2009): The study of language and space in media discourse. In: Auer, Peter/Schmidt, Jürgen E. (Hg.): Language and space: An international handbook of linguistic variation. Bd. I: Theory and methods. (= Handbücher zur Sprach- und Kommunikationswissenschaft 30,1). Berlin/New York, S. 740-758.

Androutsopoulos, Jannis (ersch.demn.): Localising the global on the participatory web. In: Coupland, Nikolas (Hg.): Handbook of language and globalization. Oxford.

Bakhtin, Mikhail (1981): Discourse in the novel. In: Bakhtin, Mikhail (Hg.): The Dialogic Imagination: 4 essays. (= University of Texas Press Slavic series 1). Austin, S. $259-422$.

Bailey, Benjamin (2007): Heteroglossia and boundaries. In: Heller, Monica (Hg.): Bilingualism: A social approach. New York, S. 257-274.

Baron, Naomi S. (2008): Always on: language in an online and mobile world. Oxford.

Birkner, Karin/Gilles, Peter (2008): Dialektstilisierung im Reality-Fernsehen. In: Christen, Helen/Ziegler, Evelyn (Hg.): Sprechen, Schreiben, Hören. Zur Produktion und Perzeption von Dialekt und Standardsprache zu Beginn des 21. Jahrhunderts. Wien, S. 101-130.

boyd, danah m. (2008): Why youth (heart) social network sites: The role of networked publics in teenage social life. In: Buckingham (Hg), S. 119-142.

boyd, danah m./Ellison, Nicole B. (2007): Social network sites: Definition, history, and scholarship. In: Journal of Computer-Mediated Communication 13, 1, article 11. Internet: http://jcmc.indiana.edu/vol13/issue1/boyd.ellison.html (Stand: November 2009).

Buckingham, David (Hg) (2008): Youth, identity, and digital media. Cambridge.

Busch, Brigitta (2004): Sprachen im Disput. Medien und Öffentlichkeit in multilingualen Gesellschaften. Klagenfurt.

Chandler, Daniel (1998): Personal home pages and the construction of identities on the Web. Internet: www.aber.ac.uk/media/Documents/short/webident.html (Stand: November 2009).

Coupland, Nikolas (2007): Style. Language variation and identity. Cambridge.

Coupland, Nikolas (2009): The mediated performance of vernaculars. In: Journal of English Linguistics 37, 3, S. 284-300.

Crystal, David (2006): Language and the Internet. 2. Aufl. Cambridge.

de.wikipedia.org (2009): Web 2.0. Internet: http://de.wikipedia.org/wiki/Web_2.0 (Stand: November 2009). 
Döring, Nicola (2002): Personal home pages on the Web: A review of research. In: Journal of Computer-Mediated Communication 7, 3. Internet: http://jcmc.indiana. edu/vol7/issue3/doering.html (Stand: November 2009).

Döring, Nicola (2003): Sozialpsychologie des Internet: die Bedeutung des Internet für Kommunikationsprozesse, Identitäten, soziale Beziehungen und Gruppen. 2., vollst. überarb. u. erw. Aufl. (= Internet und Psychologie 2). Göttingen u.a.

Duranti, Alessandro (1997): Linguistic Anthropology. Cambridge.

Ebersbach, Anja/Glaser, Markus/Heigl, Richard (2008): Social web. Konstanz.

en.wikipedia.org (2009): Web 2.0. Internet: http://en.wikipedia.org/wiki/Web_2.0 (Stand: November 2009).

Fiske, John (1987): Television culture. London/New York.

Georgakopoulou, Alexandra (2003): Computer-mediated communication. In: Verschueren, Jef/Östman, Jan-Ola/Blommaert, Jan/Bulcaen, Chris (Hg.): Handbook of pragmatics (2001 installment). Amsterdam/Philadelphia, S. 1-20.

Hasebrink, Uwe/Mikos, Lothar/Prommer, Elisabeth (Hg.) (2004): Mediennutzung in konvergierenden Medienumgebungen. München.

Heidemann, Julia (2009): Online Social Networks - Ein sozialer und technischer Überblick. In: Informatik-Spektrum.

Herring, Susan C. (2001): Computer-mediated discourse. In: Schiffrin, Deborah/Tannen, Deborah/Hamilton, Heidi E. (Hg.): The handbook of discourse analysis. Malden, S. 612-634.

Herring, Susan C. (2004): Computer-mediated discourse analysis: An approach to researching online communities. In: Barab, Sasha A./Kling, Rob/Gray, James H. (Hg.): Designing for virtual communities in the service of learning. Cambridge/ New York, S. 338-376.

Herring, Susan C. (2007): A faceted classification scheme for computer-mediated discourse. In: Language@Internet, 4, article 1. Internet: www.languageatinternet.de/ articles/2007/761/index_html/ (Stand: November 2009).

Hinchcliffe, Dion (2006): The state of Web 2.0. In: Dion Hinchcliffe's Web 2.0 Blog, 2. April 2006. Internet: http://web2.socialcomputingjournal.com/the_state_of_web_20. htm (Stand: November 2009).

Honeycutt, Courtenay/Herring, Susan C. (2009): Beyond microblogging: Conversation and collaboration via Twitter. Proceedings of the Forty-Second Hawaii International Conference on System Sciences (HICSS-42). Los Alamitos. Internet: http://ella. slis.indiana.edu/ herring/honeycutt.herring.2009.pdf (Stand: Dezember 2009).

Hutchby, Ian (2001): Conversation and technology: From the telephone to the Internet. Cambridge.

Jenkins, Henry (2006): Convergence culture: Where old and new media collide. New York.

Jones, Rodney (2004): The problem of context in computer-mediated communication. In: LeVine/Scollon (Hg.), S. 20-33. 
Kelly-Holmes, Helen (2006): Multilingualism and commercial language practices on the Internet. In: Journal of Sociolinguistics 10, 4, S. 510-523.

Kress, Gunther/van Leeuwen, Theo (2001): Multimodal discourse: The modes and media of contemporary communication. London.

LeVine, Philip/Scollon, Ron (Hg.) (2004): Discourse and technology: Multimodal discourse analysis. Washington, D.C.

Maaß, Christian/Pietsch, Gotthard (2007): Web 2.0 als Mythos, Symbol und Erwartung. (= Diskussionsbeiträge der Fakultät für Wirtschaftswissenschaft der FernUniversität in Hagen 408). Internet: www.fernuni-hagen.de/www2bonsai/wiwi/forschung/beitraege/ pdf/db408.pdf (Stand: November 2009).

Markham, Annette N./Baym, Nancy (Hg.) (2009): Internet inquiry: Conversations about method. Thousand Oaks.

Meier, Stefan (i.Vorb.): ,Share your Fotos. Watch the world‘ - Zur Nutzung und Konventionalisierung von Fotografie als Mittel visueller Imagekonstruktion im Social Web. In: Hess-Lüttich, Ernest W. B./Kimminich, Eva/Sachs-Hombach, Klaus/ Wenz, Karin (Hg.): Zeichenmaterialität, Körpersinn und (sub)kulturelle Identität, Kodikas/Code. Ars Semeiotica. Tübingen.

O'Reilly, Tim (2005): What is Web 2.0 - design patterns and business models for the next generation of software. Internet: www.oreillynet.com/pub/a/oreilly/tim/news/2005 /09/30/what-is-web-20.html (Stand: November 2009).

Paolillo, John (1999): The virtual speech community: Social network and language variation on IRC. In: Journal of Computer-Mediated Communication 4, 4. Internet: http://jcmc.indiana.edu/vol4/issue4/paolillo.html (Stand: November 2009).

Randow, Gero von (2007): Leben im Netz. In: Die Zeit, 18.01.2007. Internet: www.zeit. de/2007/04/01-Leben-im-Netz?page=all (Stand: November 2009).

Rowe, Charley/Wyss, Eva L. (Hg.) (2009): Language and new media: Linguistic, cultural, and technical evolutions. Cresskill.

Runkehl, Jens/Schlobinski, Peter/Siever, Torsten (1998): Sprache und Kommunikation im Internet: Überblick und Analysen. Opladen.

Sandig, Barbara (2006): Textstilistik des Deutschen. 2., völlig neu bearb. u. erw. Aufl. Berlin.

Schmitz, Ulrich (2002): E-Mails kommen in die Jahre. Telefonbriefe auf dem Weg zu sprachlicher Normalität. In: Dürscheid, Christa/Ziegler, Arne (Hg.): Kommunikationsform E-Mail. (= Textsorten 7). Tübingen, S. 33-56.

Schmitz, Ulrich (2003): Lesebilder im Internet. Neue Koalitionen und Metamorphosen zwischen Text und Bild. In: Zeitschrift für Germanistik, Neue Folge XIII, 3, S. 605-628.

Schmitz, Ulrich (i.d.Bd.): Schrift an Bild im World Wide Web. Articulirte Pixel und die schweifende Unbestimmtheit des Vorstellens.

Scholz, Trebor (2008): Market ideology and the myths of Web 2.0. In: First Monday 13, 3. Internet: www.uic.edu/htbin/cgiwrap/bin/ojs/index.php/fm/article/viewArticle/2138/ 1945 (Stand: November 2009). 
Siebenhaar, Beat (2006): Code choice and code-switching in Swiss-German Internet Relay Chat rooms. In: Journal of Sociolinguistics 10, 4, S. 481-509.

Stern, Susannah (2008): Producing sites, exploring identities: Youth online authorship. In: Buckingham (Hg.), S. 95-118.

Stöckl, Hartmut (2004): Die Sprache im Bild - Das Bild in der Sprache. Zur Verknüpfung von Sprache und Bild im massenmedialen Text. (= Linguistik - Impulse \& Tendenzen 3). Berlin.

Storrer, Angelika (2000): Was ist ,hyper ${ }^{6}$ am Hypertext? In: Kallmeyer, Werner (Hg.): Sprache und neue Medien. (= Jahrbuch des Instituts für Deutsche Sprache 1999). Berlin/New York, S. 222-249.

Storrer, Angelika (2001): Getippte Gespräche oder dialogische Texte? Zur kommunikationstheoretischen Einordnung der Chat-Kommunikation. In: Lehr, Andrea et al. (Hg.): Sprache im Alltag. Beiträge zu neuen Perspektiven in der Linguistik. Berlin, S. 439-465.

Tagliamonte, Sali A./Denis, Derek (2008): ,Linguistic ruin? LOL!‘ Instant messaging and teen language. In: American Speech 83, 1, S. 3-34.

Thimm, Caja (2008): Technically-mediated interpersonal communication. In: Antos, Gerd/Ventola, Eija (Hg.): Handbook of interpersonal communication. Berlin, S. 331-354.

Tsiplakou, Stavroula (2009): Doing bilingualism: Language alternation as performative construction of online identities. In: Pragmatics 19, 3, S. 361-391.

van Leeuwen, Theo (2004): Ten reasons why linguists should pay attention to visual communication. In: LeVine/Scollon (Hg.), S. 7-19.

Zimmer, Michael (2008): Critical perspectives on Web 2.0. In: First Monday 13, 3. Internet: www.uic.edu/htbin/cgiwrap/bin/ojs/index.php/fm/issue/view/263 (Stand: November 2009). 